\title{
Distribution of visible luminescence centers in hydrogen-doped $\mathrm{ZnO}$
}

\author{
Laurent L.C. Lem, Cuong Ton-That, ${ }^{\text {a) }}$ and Matthew R. Phillips \\ Department of Physics and Advanced Materials, University of Technology Sydney, \\ Broadway, New South Wales 2007, Australia
}

(Received 13 April 2011; accepted 21 October 2011)

\begin{abstract}
$\mathrm{ZnO}$ crystals have been investigated by scanning cathodoluminescence microscopy and spectroscopy at $80 \mathrm{~K}$ following hydrogen incorporation by plasma exposure. The intensity of the $\mathrm{ZnO}$ near-band-edge (NBE) emission is greatly enhanced while the defect-related green emission is quenched following plasma treatment. These effects are attributed to the passivation of zinc vacancies by hydrogen. The green and yellow intensities and their intensity ratios to the NBE vary with excitation depth for both undoped and $\mathrm{H}$-doped $\mathrm{ZnO}$ crystals. The intensities of the green and yellow emissions exhibit sublinear dependencies on electron beam excitation density while the NBE intensity increases linearly with the excitation density. These saturation effects with increasing excitation density must be taken into account when assessing defects in $\mathrm{ZnO}$ by luminescence characterization.
\end{abstract}

\section{INTRODUCTION}

$\mathrm{ZnO}$ is a promising material for optoelectronic applications due to its large exciton binding energy $(60 \mathrm{meV})$, low refractive index (1.9), and stability under intense ultraviolet exposure. Although it is relatively simple to grow high-quality single crystal n-type $\mathrm{ZnO}$, fabricating stable p-type material is proving to be difficult despite an intense research effort. Along with native defects such as oxygen vacancy $\left(V_{\mathrm{O}}\right)$, zinc interstitial $\left(\mathrm{Zn}_{i}\right)$, and zinc vacancy $\left(V_{\mathrm{Zn}}\right)$, hydrogen has been proposed to be an important source of n-type conductivity in $\mathrm{ZnO} .{ }^{1}$ Firstprinciples investigations based on density functional theory have revealed that hydrogen in $\mathrm{ZnO}$, unlike in other semiconductors, acts exclusively as a shallow donor impurity, ${ }^{1}$ which has recently been confirmed by experiments. ${ }^{2}$ Hydrogen can also have a pronounced effect on the optical and electronic properties of $\mathrm{ZnO}^{2-4}$ especially it has a very high diffusivity in $\mathrm{ZnO}$ and is a common impurity usually present during the processing of $\mathrm{ZnO}$ crystals. ${ }^{5}$ Understanding the properties of hydrogen in $\mathrm{ZnO}$ is pivotal to lowering the background n-type conductivity levels and improving the possibility of producing p-type $\mathrm{ZnO}$.

It has been reported that the near-band-edge (NBE) luminescence efficiency in $\mathrm{ZnO}$ is significantly enhanced by the incorporation of hydrogen, ${ }^{6,7}$ but there is little systematic data available on its effects on defect-related visible emissions. Two commonly observed defect-related emissions in $\mathrm{ZnO}$ are: (i) ubiquitous green luminescence that has been assigned to native defects ${ }^{8,9}$ and (ii) yellow luminescence attributed to the presence of $\mathrm{Al}$ or $\mathrm{Li}$ impurities. ${ }^{10}$ The aim of the present study is to evaluate the role of

\footnotetext{
a)Address all correspondence to this author.

e-mail: Cuong.Ton-That@uts.edu.au

DOI: $10.1557 /$ jmr.2011.383
}

hydrogen incorporation in the defect-related and NBE emissions in $\mathrm{ZnO}$. Cathodoluminescence (CL) in a scanning electron microscope (SEM) is a powerful technique used extensively to study structural defects and the optical properties of semiconductor materials and devices. ${ }^{11}$ The depth profile of a particular luminescence center can be obtained nondestructively by measuring CL spectra as a function of the electron penetration range. This CL method is conducted by changing the electron beam energy while adjusting the beam current to maintain the beam power and hence the electron-hole pair injection rate. ${ }^{12}$ In this way, the in-depth distribution of luminescence centers in a specimen can be analyzed. In this work, we show that the green and yellow emissions exhibit different behaviors upon hydrogen incorporation and different dependencies on the photon generation depth. Understanding these variations is vital for a correct interpretation of luminescence data from $\mathrm{ZnO}$.

\section{EXPERIMENTAL}

$\mathrm{ZnO}$ crystals used in this work were hydrothermally grown by the MTI Corporation. Samples (MTI Corp., Richmond, CA) were polished both sides, $0.5 \mathrm{~mm}$ thick, $1 \mathrm{~cm} \times 1 \mathrm{~cm}$ square sections. The normally undoped $a$-plane crystal (resistivity $160 \Omega \cdot \mathrm{cm}$ ) was exposed to a hydrogen radio-frequency plasma of power $15 \mathrm{~mW}$ for $10 \mathrm{~min}$ at $200{ }^{\circ} \mathrm{C}$. Under these conditions, the incorporation depth of hydrogen is expected to be $>10 \mu \mathrm{m} .{ }^{4}$ No morphological changes due to the plasma treatment were detected by SEM observation. CL was performed at $80 \mathrm{~K}$ using a Quanta 200 SEM (FEI Corp., Hillsboro, OR) equipped with a liquid nitrogen cold stage and an Ocean Optics SD2000 (Dunedin, FL) Diode Array Spectrometer. CL measurements were performed by two methods: (i) changing the beam accelerating voltage with a constant beam power to probe the in-depth distribution of luminescence centers in the crystal 
and (ii) changing the electron beam current while maintaining the accelerating voltage to analyze excitation density dependencies of particular emissions. All collected data was corrected for the response of the system.

\section{RESULTS AND DISCUSSION}

Figure 1 shows the $\mathrm{CL}$ spectra of the $\mathrm{ZnO}$ crystal before and after hydrogen incorporation. These spectra, collected from a $5 \mu \mathrm{m} \times 5 \mu \mathrm{m}$ scan region at $80 \mathrm{~K}$ with the same resolution and the SEM operated at $20 \mathrm{kV}$ and $1.3 \mathrm{nA}$, display similar spectral features in the NBE region for the undoped and H-doped crystals. The NBE CL consists of a donor bound exciton $\left(\mathrm{D}^{0} \mathrm{X}\right)$ emission overlapped with free exciton (FX) emission at $3.37 \mathrm{eV}$, along with $\mathrm{FX}$ replicas (FX-1LO, FX-2LO, and FX-3LO) separated by an equal energy space of the longitudinal optical-phonon energy $E_{\mathrm{LO}}=72 \mathrm{eV} \cdot{ }^{13,14}$ Upon hydrogen incorporation, the NBE intensity increases by almost an order of magnitude. The high energy side of the peak is enhanced only moderately due to the effect of optical self-absorption. Monochromatic CL images taken at $3.37 \mathrm{eV}$ reveal uniform emission from the crystal surface, indicating a high degree of the uniformity of hydrogen incorporation.

A broad defect-related visible band is observed in both undoped and $\mathrm{H}$-doped crystals. While the peak position of the NBE was unchanged following the hydrogen incorporation, the defect band not only strongly red shifts but also has a significant reduction in intensity. This band can be fitted by two Gaussian components centered at $2.1 \mathrm{eV}$ (yellow) and $2.5 \mathrm{eV}$ (green) (Fig. 2), which have been recently attributed to radiative recombination involving lithium acceptor ${ }^{10,15,16}$ and centers involving zinc/oxygen

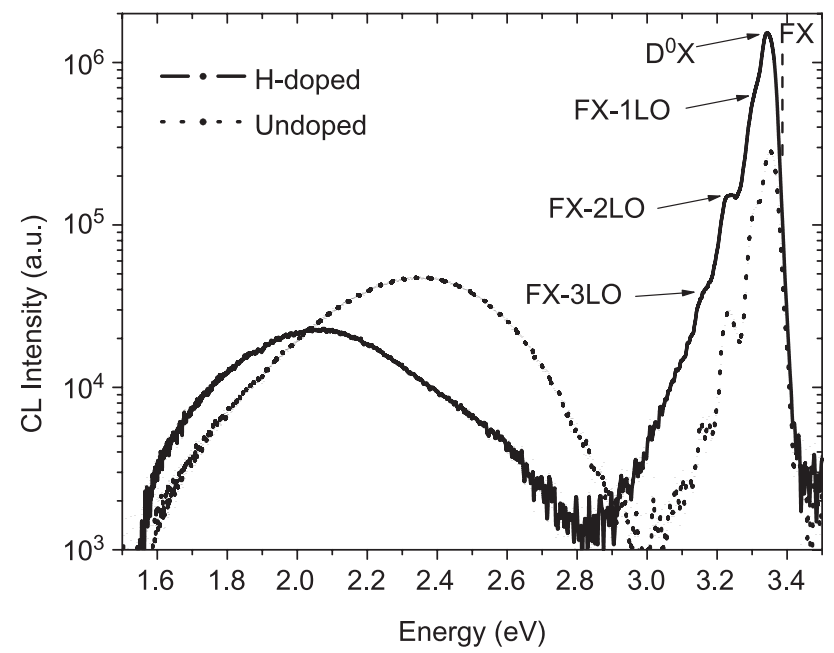

FIG. 1. Cathodoluminescence (CL) spectra obtained at $80 \mathrm{~K}$ from the $\mathrm{ZnO}$ crystal (electron beam energy $=20 \mathrm{keV}$, beam current $=1.3 \mathrm{nA}$ ) before and after hydrogen plasma treatment. The near-band-edge (NBE) intensity increases by a factor of 10 following hydrogen incorporation while the defect-related emission is quenched. vacancies, ${ }^{8,17}$ respectively. Hydrothermally grown $\mathrm{ZnO}$, synthesized in a solution containing $\mathrm{LiOH}$, is known to have $\mathrm{Li}$-related defects, which are responsible for the yellow luminescence. ${ }^{15}$ The observed red shift is due to variations in the relative intensities of the yellow and green emissions. These results are illustrated more fully in Fig. 3, which presents the depth-resolved CL results for these two components as well as for the NBE. This figure also contains a secondary $x$-axis for the CL generation depth corresponding to the distance over which $70 \%$ of the CL intensity is generated. These data were obtained from simulations of 5000 trajectories of electrons in $\mathrm{ZnO}$ using the Monte Carlo stimulation CASINO. ${ }^{18}$ (In addition to the spatial spread of the injected electrons, the diffusion of carriers was also considered for the determination of the CL generation depth.) The green emission is dominant throughout the generation depth up to $1500 \mathrm{~nm}$ for the undoped sample but quenched considerably following hydrogen incorporation. The yellow defect emission is slightly enhanced at depths greater than about $300 \mathrm{~nm}$. The reduction in the green intensity is fairly constant within in the depth range $300-2100 \mathrm{~nm}$, indicating a uniform hydrogen concentration, in good agreement with depth profiling measurements. ${ }^{4}$

The decreases in the intensity of the NBE emission at depths greater than $250 \mathrm{~nm}$ for the undoped crystal and $1200 \mathrm{~nm}$ for the H-doped crystal are due to strong selfabsorption of the emitted photons by $\mathrm{ZnO}$. The NBE intensity is attenuated by $\exp (-\alpha \cdot d)$, where $\alpha$ is the absorption coefficient and $d$ is the photon generation depth. Figure 4 represents the fit to the NBE intensity data of the undoped crystal with $d$ being taken as the CL generation depth, yielding $\alpha=(2.8 \pm 0.4) \times 10^{3} \mathrm{~cm}^{-1}$, which is in good agreement with the previously measured value for $3.3 \mathrm{eV}$ photons by ultraviolet-visible absorption spectroscopy. ${ }^{19}$ It is worth noting that accurate determination of the

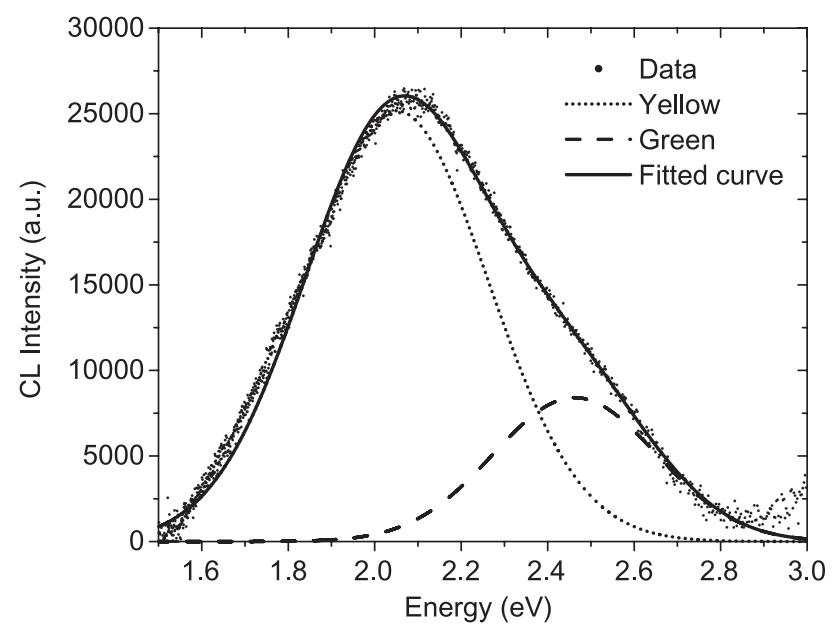

FIG. 2. The broad defect-related visible band of $\mathrm{H}$-doped $\mathrm{ZnO}$ following plasma treatment in Fig. 1 can be fitted with two Gaussian components centered at $2.1 \mathrm{eV}$ (yellow) and $2.4 \mathrm{eV}$ (green), which are related to $\mathrm{Li}$ impurity and native defects, respectively. 
absorption coefficient is not possible from depth-resolved CL since photons are generated up to the electron range. The weak luminescence in the near-surface region $(<250 \mathrm{~nm})$ is due to nonradiative recombination centers created by surface polishing. ${ }^{20}$ These results show that the NBE emission is only detected close to the surface, whereas the green and yellow emissions can originate from much greater depths (micron scale) in the crystal because such photons are not absorbed.

The considerable increase in the NBE intensity following hydrogen incorporation can be attributed to the introduction of an efficient radiative recombination pathway, which mainly arises from hydrogen shallow donors located at the bond-centered site $\left(\mathrm{H}_{\mathrm{BC}}\right) .{ }^{1}$ This explanation

\section{Generation Depth (nm)}

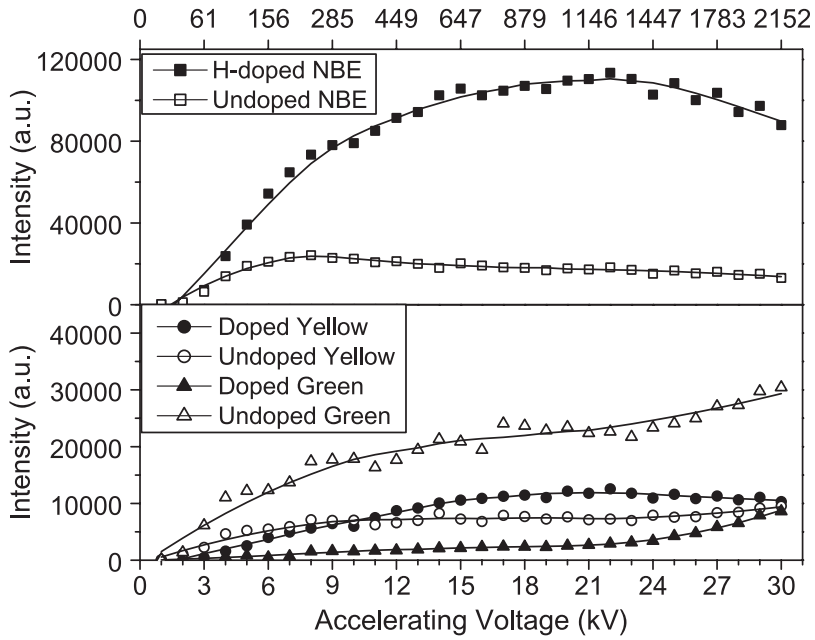

FIG. 3. Integrated intensities of the NBE, yellow, and green luminescence as a function of electron beam energy obtained at $80 \mathrm{~K}$. The beam current, $I_{\mathrm{B}}$, was adjusted to provide a constant beam power of $70 \mu \mathrm{W}$. Also shown is the luminescence generation depth in $\mathrm{ZnO}$ corresponding to $70 \% \mathrm{CL}$ intensity obtained by Monte Carlo simulations.

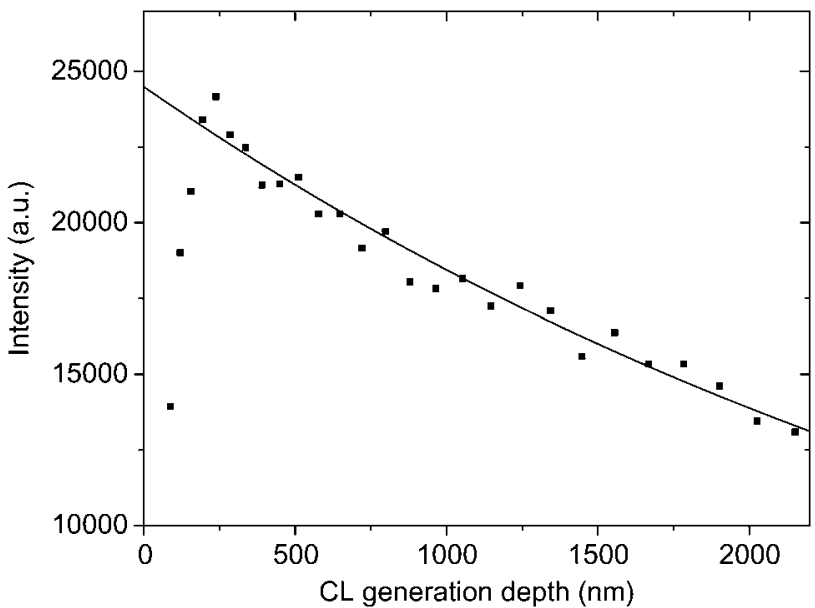

FIG. 4. For depths $>250 \mathrm{~nm}$, the NBE intensity of the untreated crystal can be fitted by an exponential function, which yields the absorption coefficient $\alpha=(2.8 \pm 0.4) \times 10^{3} \mathrm{~cm}^{-1}$. is supported by Hall-effect measurements, which show the carrier density increases from $2.0 \times 10^{14}$ to $1.7 \times 10^{17} \mathrm{~cm}^{3}$ upon plasma treatment. The elimination of competitive radiative recombination channels as evidenced by the dramatic reduction of the green emission will also contribute to the increase in the NBE emission and yellow luminescence. It is worth noting that the treatment temperature $200{ }^{\circ} \mathrm{C}$ is not sufficient to introduce more defects in the crystal since the thermal energy is considerably smaller than the formation energies of intrinsic point defects in $\mathrm{ZnO} .^{8}$ Defect centers involving surface oxygen and bulk zinc vacancies are the most likely candidates for the green luminescence as they are theoretically predicted to have the lowest formation energy and should be the most abundant native defects. ${ }^{8,21}$ However, the reduction of the green emission from the bulk of the crystal following hydrogen incorporation demonstrates that it cannot originate from surface oxygen vacancies. On the other hand, zinc vacancies are double acceptors and occur in the 2-charge state $\left(V_{\mathrm{Zn}}{ }^{2-}\right)$. Hydrogen, known to act almost exclusively as positive charge state $\left(\mathrm{H}^{+}\right),{ }^{1}$ would preferentially interact with $V_{\mathrm{Zn}}{ }^{2-}$ rather than $\mathrm{V}_{\mathrm{O}}^{+}$or $\mathrm{V}_{\mathrm{O}}^{++}$centers. This interpretation is in agreement with first-principles calculations, which indicate the existence of a complex involving $V_{\mathrm{Zn}}{ }^{2-}$ and two hydrogen atoms. ${ }^{22}$ This explanation is further supported by the stability of hydrogen in the $\mathrm{ZnO}$ crystal as evidenced by the fact that the $\mathrm{CL}$ spectrum of the hydrogen-doped $\mathrm{ZnO}$ crystal remains unchanged 6 weeks after plasma treatment.

Figure 5 shows the variations of the NBE, yellow, and green intensities on the beam current over a scan area of

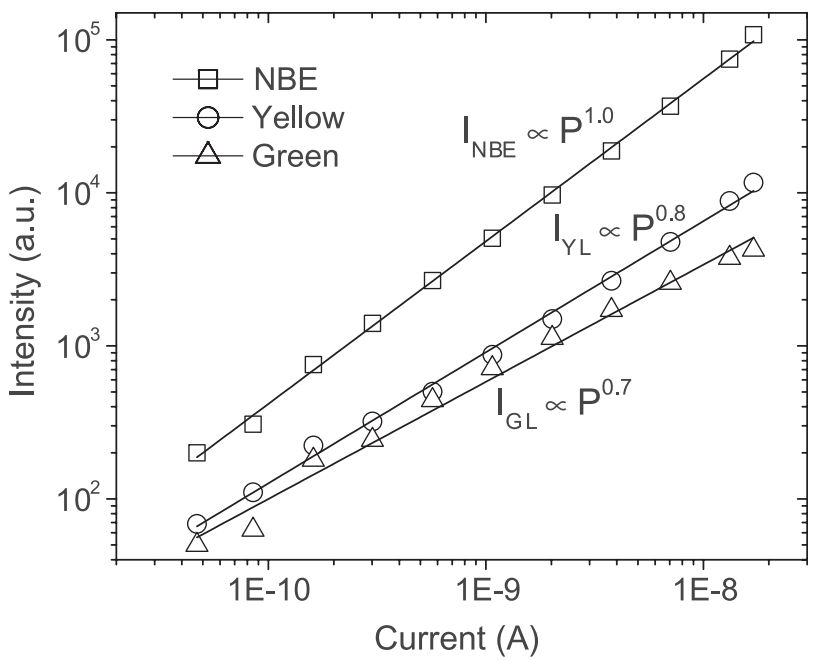

FIG. 5. Intensities of the NBE, green, and yellow emissions at $80 \mathrm{~K}$ as a function of electron beam current obtained with beam energy of $30 \mathrm{keV}$. Power-law fits by straight lines show variations with the beam current $\left(I_{\mathrm{B}}\right): I_{\mathrm{NBE}} \propto I_{\mathrm{B}}{ }^{1}, I_{\mathrm{GL}} \propto I_{\mathrm{B}}{ }^{0.7}, I_{\mathrm{YL}} \propto I_{\mathrm{B}}{ }^{0.8}$. The power-law exponents indicate that the yellow and green emission peaks saturate with increasing $I_{\mathrm{B}}$, where as the NBE has a linear dependence consistent with an excitonic emission. 
a $5 \mu \mathrm{m} \times 5 \mu \mathrm{m}$. In this measurement, the accelerating voltage was fixed at $30 \mathrm{kV}$, while the beam current was varied from $50 \mathrm{pA}$ to $20 \mathrm{nA}$. A power-law model $\left(I_{\mathrm{CL}} \propto I_{\mathrm{B}}{ }^{\mathrm{m}}\right)$, where $I_{\mathrm{CL}}$ is the peak intensity and $I_{\mathrm{B}}$ the beam current, is used to evaluate the excitation power dependence of the CL intensity. Power-law fits, shown by straight lines in the $I_{\mathrm{CL}}$ versus $I_{\mathrm{B}} \log -\log$ plots, reveal that the green and yellow intensities exhibit strongly sublinear behaviors, with $I_{\text {yellow }} \propto I_{\mathrm{B}}{ }^{0.8}$ and $I_{\text {green }} \propto I_{\mathrm{B}}{ }^{0.7}$. The dependence of the NBE on beam current is linear, $I_{\mathrm{NBE}} \propto I_{\mathrm{B}}$. The strongly sublinear dependencies of the yellow and green emissions are not unexpected and characteristic of recombination channels involving deep levels. This sublinear behavior of CL spectra discussed here has been observed for other semiconductors and interpreted in terms of saturation kinetics. ${ }^{23}$ While the ratio of the NBE-to-defect emissions is frequently used to determine the quality of $\mathrm{ZnO}$, this nonlinearity with electron beam density must be taken into account for a correct interpretation of $\mathrm{CL}$ data from $\mathrm{ZnO}$.

\section{CONCLUSION}

In conclusion, depth-resolved CL spectra of $\mathrm{ZnO}$ crystals were investigated under different accelerating voltages and beam currents before and after hydrogen plasma treatment. The green and yellow intensities and its intensity ratio to the NBE vary with excitation depth for both undoped and $\mathrm{H}$-doped $\mathrm{ZnO}$ specimens. From the comparison with the CL spectra before and after hydrogen incorporation, it is strongly suggested that the quenching of the green luminescence is due to the formation of nonradiative defect complexes involving zinc vacancies and hydrogen.

\section{ACKNOWLEDGMENT}

This work was supported by the Australian Research Council.

\section{REFERENCES}

1. C.G. Van de Walle: Hydrogen as a cause of doping in zinc oxide. Phys. Rev. Lett. 85, 1012 (2000).

2. Y.M. Strzhemechny, H.L. Mosbacker, D.C. Look, D.C. Reynolds, C.W. Litton, N.Y. Garces, N.C. Giles, L.E. Halliburton, S. Niki, and L.J. Brillson: Remote hydrogen plasma doping of single crystal ZnO. Appl. Phys. Lett. 84, 2545 (2004).

3. Y.M. Strzhemechny, J. Nemergut, P.E. Smith, J. Bae, D.C. Look, and L.J. Brillson: Remote hydrogen plasma processing of $\mathrm{ZnO}$ single crystal surfaces. J. Appl. Phys. 94, 4256 (2003).

4. K. Ip, M.E. Overberg, Y.W. Heo, D.P. Norton, S.J. Pearton, C.E. Stutz, S.O. Kucheyev, C. Jagadish, J.S. Williams, B. Luo, F. Ren, D.C. Look, and J.M. Zavada: Hydrogen incorporation, diffusivity and evolution in bulk $\mathrm{ZnO}$. Solid-State Electron. 47, 2255 (2003).

5. C. Jagadish and S.J. Pearton: Zinc Oxide bulk, thin films and nanostructures: Processing, properties and applications (Elsevier, New York, 2006).

6. T. Sekiguchi, N. Ohashi, and Y. Terada: Effect of hydrogenation on ZnO luminescence. Jpn. J. Appl. Phys. 36, L289 (1997).

7. N. Ohashi, T. Ishigaki, N. Okada, T. Sekiguchi, I. Sakaguchi, and H. Haneda: Effect of hydrogen doping on ultraviolet emission spectra of various types of ZnO. Appl. Phys. Lett. 80, 2869 (2002).

8. A. Janotti and C.G. Van de Walle: Native point defects in $\mathrm{ZnO}$. Phys. Rev. B 76, 165202 (2007).

9. C. Ton-That, M.R. Phillips, M. Foley, S.J. Moody, and A.P.J. Stampfl: Surface electronic properties of $\mathrm{ZnO}$ nanoparticles. Appl. Phys. Lett. 92, 261916 (2008).

10. J. Nayak, S. Kimura, S. Nozaki, H. Ono, and K. Uchida: Yellowishwhite photoluminescence from $\mathrm{ZnO}$ nanoparticles doped with $\mathrm{Al}$ and Li. Superlattices Microstruct. 42, 438 (2007).

11. B.G. Yacobi and D.B. Holt: Cathodoluminescence microscopy of inorganic solids (Plenum, New York, 1990).

12. S.M. Davidson and C.A. Dimitriadis: Advances in the electrical assessment of semiconductors using the scanning electron microscope. J. Microsc. 118, 275 (1980).

13. M.R. Phillips, O. Gelhausen, and E.M. Goldys: Cathodoluminescence properties of zinc oxide nanoparticles. Phys. Status Solidi A 201, 229 (2004).

14. L.J. Wang and N.C. Giles: Temperature dependence of the freeexciton transition energy in zinc oxide by photoluminescence excitation spectroscopy. J. Appl. Phys. 94, 973 (2003).

15. T.M. Borseth, B.G. Svensson, A.Y. Kuznetsov, P. Klason, Q.X. Zhao, and M. Willander: Identification of oxygen and zinc vacancy optical signals in ZnO. Appl. Phys. Lett. 89, 262112 (2006).

16. T. Sekiguchi, S. Miyashita, K. Obara, T. Shishido, and N. Sakagami: Hydrothermal growth of $\mathrm{ZnO}$ single crystals and their optical characterization. J. Cryst. Growth 214, 72 (2000).

17. K. Vanheusden, W.L. Warren, C.H. Seager, D.R. Tallant, J.A. Voigt, and B.E. Gnade: Mechanisms behind green photoluminescence in ZnO phosphor powders. J. Appl. Phys. 79, 7983 (1996).

18. D. Drouin, A.R. Couture, D. Joly, X. Tastet, V. Aimez, and R. Gauvin: CASINO V2.42-A fast and easy-to-use modeling tool for scanning electron microscopy and microanalysis users. Scanning 29, 92 (2007)

19. A. Nadarajah and R. Konenkamp: Laser annealing of photoluminescent $\mathrm{ZnO}$ nanorods grown at low temperature. Nanotechnology 22, 025205 (2011).

20. J. Mass, M. Avella, J. Jimenez, M. Callahan, E. Grant, K. Rakes, D. Bliss, and B.G. Wang: Cathodoluminescence characterization of hydrothermal ZnO crystals. Superlattices Microstruct. 38, 223 (2005).

21. J.D. Prades, A. Cirera, J.R. Morante, and A. Comet: Ab initio insights into the visible luminescent properties of $\mathrm{ZnO}$. Thin Solid Films 515, 8670 (2007).

22. E.V. Lavrov, J. Weber, F. Borrnert, C.G. Van de Walle, and R. Helbig: Hydrogen-related defects in $\mathrm{ZnO}$ studied by infrared absorption spectroscopy. Phys. Rev. B 66, 165205 (2002).

23. S.O. Kucheyev, M. Toth, M.R. Phillips, J.S. Williams, and C. Jagadish: Effects of excitation density on cathodoluminescence from GaN. Appl. Phys. Lett. 79, 2154 (2001). 